\title{
Zusammenfassende Betrachtung über den Inhalt der vorhergehenden Abhandlungen.
}

\author{
Von
}

K. Hürthle.

Die Veranlassung zur Anstellung der vorliegenden Untersuchungen bildete der Vergleich der Druck- und Strompulse von Karotis und Cruralis beim Hunde. Dabei zeigte sich eine unerklärliche Störung der sonst vorhandenen Beziehung zwischen Druck und Stromstärke, die als systolische Schwellung des Stromes (s S) bezeichnet wurde und zur Erörterung der Frage fübrte, ob die Arterien aktiv an der Bewegung des Blutes beteiligt seien, etwa in der Weise, dass sie der Sitz peristaltischer Wellen sind, die durch den Puls ausgelöst, über das Arteriensystem ablaufen und eine systolische Beschleunigung des Stromes durch Arbeitsleistung oder durch Herabsetzung der Widerstände bewirken. Diese Hypothese wird zwar schon beute von mancher Seite ${ }^{1}$ ) als zutreffend betrachtet, ist aber nach meiner Ansicht durchaus nicht einwandfrei begründet ${ }^{2}$ ). Von anderer Seite ${ }^{3}$ ) wird die Hypothese als unwahrscheinlich abgelehnt. Die Schwierigkeit liegt in der Beibringung entscheidender Tatsachen. Der durch den Vergleich der Druck- und Strompulse gegebene Anhaltspunkt wurde mit der Überlegung weiter verfolgt, dass, wenn die $\mathrm{S} S$ die Wirkung einer aktiven Tätigkeit der Arterien ist, es gelingen müsste, sie durch erregende Mittel zu verstärken und durch lähmende aufzuheben.

Da der quantitative Vergleich der Druck- und Strompulse die Kenntnis der beteiligten Faktoren und ihrer funktionellen Beziehungen zur Voraussetzung bat, wurde zunächst die schon früher begründete

1) K. Hasebroek, Über den extrakardialen Kreislauf des Blutes usw. Jena 1914.

2) K. Hürthle, Kritischer Bericht über das Buch von K. Hasebroek. Berliner klin. Wochenschr. 1914 Nr. 30.

3) C. Hess, Über die funktionelle Bedeutung der Arterienmuskulatur. Korrespbl. f. Schweizer Ärzte 1914 Nr. 32. 
Analyse der Kurven nach dem Windkesselprinzip einer erneuten Prüfung unterzogen (Abhandlung I). Dazu dienten Versuche an einem Schema, das die nach unserem heutigen Wissen wesentlichen Eigenschaften der arteriellen Bahn enthält und Strom- und Druckkurven liefert, für welche die mitwirkenden Faktoren bekannt sind. Der Grad der Übereinstimmung der registrierten mit den nach der Theorie berechneten Stromkurven bildet das Mass für die Zulässigkeit der Theorie und für die Fehler der Methode. Der Vergleich ergab, dass die Übereinstimmung zwischen den beiden Kurven eine um so vollständigere ist, je geringer die Dehnbarkeit der zwischen Stromuhr und Kapillaren liegenden Bahnstrecke - des peripheren Windkessels $(\mathrm{p} W)$ - ist. Ist diese verschwindend klein, so gehen die Abweichungen nicht über 5-10\%. Erreicht die Dehnbarkeit einen Wert, der wenigstens zehnmal grösser ist als der der Bahn der Cruralis eines mittelgrossen Hundes, so gehen die Abweichungen bis $20 \%$, jedoch nur im Verlauf des aufsteigenden Schenkels der Druckkurve, während im absteigenden auch hier $10 \%$ Fehler nicht erreicht werden.

Da bei den Tierpulsen von Karotis und Cruralis der $p \mathrm{~W}$ sehr klein ist (Abhandlung IV S. 365), muss erwartet werden, dass bei diesen die Abweichungen der registrierten von den berechneten Stromkurven auch im systolischen Teil 10\% nicht wesentlich überschreiten, falls hier dieselben Faktoren massgebend sind wie im Schema.

Tatsäehlich erreichten die Abweichungen in mehreren Versuchsreihen im höchsten Falle $11 \%$ und schwankten meist um 5\%, so dass man die Übertragung der Theorie auf die Blutbahn prinzipiell als zulässig bezeichnen kann. Dieses Ergebnis wurde aber nicht an den Pulsen der normalen Blutbahn gewonnen, sondern nur nach schädigenden Eingriffen, infolge deren die Gefässe als gelähmt betrachtet werden dürfen. Unter diesen Bedingungen wird die s $\mathrm{S}$ an der Stromkurve vermisst.

Im Gegensatz dazu lässt sich eine ausserordentliche Verstärkung der an normalen Pulsen auftretenden $\mathrm{S}$ durch erregende Mittel (Adrenalin, Pituitrin, Digitalis) erzielen (Abhandlung III). Auf der Höhe der Wirkung werden die Abweichungen der registrierten von der berechneten Kurve so gross, dass sie $100 \%$ und darüber betragen. Beim „zweiten Grad" der Adrenalinwirkung findet Strömung nur im systolischen Teil des Pulses 
statt, im diastolischen ist sie gleich Null, beim dritten Grad wird der diastolische Strom sogar rückläufig.

Durch die Versuche mit Lähmung und Erregung der Gefässe wird also die Frage nach der physikalischen oder physiologischen Naturder s S dahin beantwortet, dass nach Lähmung der Gefässe für den Verlauf der Strompulse dieselben Faktoren massgebend sind wie im Schema, dass aber schon unter normalen Umständen und noch mehr nach künstlicher Erregung der Gefässe eine solche Störung der Beziehung $z$ wischen Druck und Stromstärke eintritt, dass die Mitwirkung eines weiteren, im Schemanicht berücksichtigten Faktors angenommen werden muss.

Worin besteht dieser Faktor?

Zunächst lässt sicb feststellen, dass er nur bei der pulsatorischen Tätigkeit auftritt und zu einer Beschleunigung des systolischen Stromes führt. Das geht zunächst aus den Versuchen von F. Schaefer (Abbandlung V) über den Unterschied der Wirkung des konstanten und rhythmischen Druckes hervor. In diesen hat sich gezeigt, dass die bei künstlicher Durchströmung durch die Hinterbeine des Frosches laufenden Flüssigkeitsmengen bei gleichen Werten des Mitteldruckes gleich sind, sei es, dass der Druck konstant ist oder pulsatorische Schwankungen zeigt. Dieses Verhalten ändert sich aber bei der Anwendung gefässerregender Mittel (Adrenalin, Pituitrin, Digitalis). derart, dass die Stromstärken bei der rhythmischen Durchströmung um ein Drittel bis die Hälfte grösser werden als bei der konstanten.

Zweitens: Während beim Froseh die beschleunigende Wirkung der Systole nur unter dem Einfluss vasomotorischer Mittel zum Vorschein kommt, zeigt sie sich beim Hunde schon bei normalen Gefässen im Strompuls in Form der s S und noch ausgeprägter im folgenden Falle: Wenn während einer durch Vagusreizung erzeugten anhaltenden Diastole unvollständige Systolen auftreten, die zu geringen Steigerungen des absinkenden Druckes führen, so bewirken diese Beschleunigungen des Stromes, die in keinem Verhältnis zum Mitteldruck stehen, sondern erheblich grösser sind (Abhandlung II S. 336). Dass unter der Anwendnng gefässerregender Mittel beim Hunde der fördernde Einfluss der Systole noch stärker als beim Frosch hervortritt, lehrt die Adrenalinwirkung. Aus diesen Versuchen geht hervor, dass unter dem Einfluss der Drucksehwankungen 
ein für die Strömung wichtiger Faktor auftritt, der beim konstanten Druck fehlt.

Die Natur des fraglichen Faktors ist noch nicht aufgeklärt. Die in dieser Richtung angestellten Versuche (künstliche Durchströmung der Hinterbeine mit Blut bzw. Ringer-Lösung S. 366) weisen zwar darauf bin, dass die geformten Elemente des Blutes den Verlauf der Strompulse erheblich zu beeinflussen vermögen, sofern mit dem Wechsel des Tonus der Radienquotient $(\mathrm{R}-\mathrm{Q}=$ Verhältnis von Körperchen- und Röhrendurchmesser S. 330) und damit die Reibung des Blutes sowie ihre Abhängigkeit vom Druck sich erheblich ändert. Doch ist es bisher nicht gelungen, die verschiedenen Formen der Strompulse auf diese oder eine andere physikalische Ursache zurückzuführen, weil der Einfluss des R-Q während des steigenden und sinkenden Druckes sich in gleicher Weise geltend machen muss, und eine Beschränkung der Strömung auf die erste Phase damit nicht erklärt werden kann.

Wären die starken Schwankungen im Verhältnis der diastolischen zur systolischen Stromstärke dem Einfluss des R-Q zuzuschreiben, so wäre zu erwarten, dass sie dem Tonus der Gefässe parallel gehen. Das trifft zwar im allgemeinen zu, ist aber nicht streng der Fall. Unter den erregenden Mitteln nimmt das Kalziumchlorid eine Ausnahmestellung ein, unter den lähmenden das Yobimbin (Abhandlung III S. 354; Abhandlung IV S. 374). Auch in den Versuchen von Fr. Schaefer über den Einfluss des konstanten und rhythmischem Druckes auf die mittlere Stromstärke zeigte sich, dass die fördernde Wirkung des Pulses, welche bei Erregung der Gefässe durch Adrenalin festzustellen ist, nicht an die konstriktorische gebunden ist; vielmehr konnten zwei Gruppen von gefässerregenden Mitteln untersebieden werden: Die erste Gruppe, zu der Adrenalin, Pituitrin und Digitalis gehören, bewirkt zugleich mit der Herabsetzung der mittleren Stromstärke eine pulsatorische Förderung des Stromes, die zweite Gruppe, in der sich Kalziumchlorid, Nikotin und Strychnin finden, setzt die mittlere Stromstärke herab, obne sie beim rhythmischen Druck stärker zu machen als beim konstanten.

Diese Erscheinungen setzen einer physikalischen Erklärung grosse Schwierigkeiten entgegen, weil es nicht möglich ist, den Unterschied der beiden Gruppen vor vasomotorischen Mitteln dem Einfluss des R-Q zuzuschreiben. Wäre dieser für die eigentümliche Wirkung des Adrenalins verantwortlich, so müsste er es auch für 
die des Kalziumchlorids sein. Dazu kommt, dass sich die s S durch Adrenalin auch bei künstlichen Pulsen mit körperchenfreier Flüssigkeit (Ring er-Lösung) erzielen lässt (Abhandlung IV S. 368).

Wir werden somit auf die physiologische Natur des fraglichen Faktors hingewiesen. Das Wesen des Faktors entzieht sich aber vorläufig unserer Kenntnis. Der Hypothese der peristaltischen Tätigkeit der Arterien stellen sich nicht unerhebliche Schwierigkeiten entgegen. Das von C. $\mathrm{Hess}^{1}$ ) geäusserte Bedenken, dass im Gefässsystem keine Einrichtungen vorhanden sind, welche den durch eine peristaltische Welle erzeugten Druck in ei ner Richtung zur Wirkung kommen lassen würden, kann ich zwar nicht als Beweis gegen die Wirksamkeit einer peristaltischen Welle anerkennen, da es denkbar ist, dass die Welle durch ibre Form Wirkungen in einer Richtung ausübt; ich erinnere nur an die. Flimmerhaare, die durch schwingende Bewegung einseitige Wirkungen erzielen.

Auch das Bedenken, dass zur Erklärung einer peristaltischen Welle der Gefässmuskulatur Eigenschaften (bezüglich der Latenz, der Dauer der Verkürzung und der Fortpflanzungsgeschwindigkeit der Erregung) zugeschrieben werden müssten, die wir an der übrigen glatten Muskulatur der Wirbeltiere nicht kennen, ist kein ernstlicher Grund gegen die Hypothese, da es möglich ist, dass die Gefässmuskulatur besondere Eigenschaften besitzt.

Neuerdings ist in den rhythmisch-automatischen Kontraktionen überlebender Arterien eine einwandfreie Tatsache für das Vorkommen aktiver Tätigkeit der Arterien festgestellt worden ${ }^{2}$ ). Die Kontraktionen werden unter dem Reiz eines gleichförmigen Druckes (anhaltender Belastung) ausgelöst und unterhalten und bestehen in einer regelmässig sich wiederholenden Kontraktion und Erschlaffung der Wand. Als Beweis für unsere Hypothese können aber diese Bewegnngen nicht angesehen werden, weil ihr zeitlicher Verlauf ein äusserst langsamer, nämlich von der Ordnung ist, welche den glatten Muskeln der Warmblüter gewöhnlich zukommt. Zur Stütze einer peristaltischen Tätigkeit der Arterien

1) C. Hess, I. c.

2) 0. B. Meyer, Über einige Eigenschaften der Gefässmuskulatur mit besonderer Berücksichtigung der Adrenalinwirkang. Zeitschr. f. Biol. Bd. 48 S. 352. 1906. - Hermann Full, Versuche über die automatischen Bewegungen der Arterien. Ebenda Bd. 61 S. 287. 1913. - Gustav Günther, Zur Kenntnis der Spontanbewegung überlebender Arterien. Ebenda Bd. 65 S. 401. 1915. 
müssten aber zuckungsartige Reaktionen der Arterien beobachtet werden von einer den Skelettmuskeln eigenen Geschwindigkeit des Verlaufs.

Was mich aber am Vorkommen einer Arterienperistaltik immer noch zweifeln lässt, ist der Umstand, dass verschiedene Versuche zum direkten Nachweis der Erscheinung bisher nicht zum Ziele geführt haben:

Zwar schien der Nachweis pulsatorischer Aktionsströme ${ }^{1}$ ) der Arterien auf den ersten Anlauf geglückt zu sein, aber die weiteren, in der Abhandlung VI von Blumenfeld t beschriebenen Versuche lassen keinen $\mathrm{Zweifel} \mathrm{darüber,} \mathrm{dass} \mathrm{die} \mathrm{bei} \mathrm{pulsatorischer} \mathrm{Durch-}$ strömung von Arterien auftretenden elektrischen Vorgänge im wesentlichen physikalischer Natur (Strömungsströme) sind. Nur an den Arterien der Nabelschnur zeigen sich neben den Strömungsströmen elektrische Schwingungen, die als Aktionsströme gedeutet werden können. Es besteht daher immer noch die Möglichkeit, dass letztere auch bei den Körperarterien vorkommen, und dass sie nur von einer Grössenordnung sind, welche gegen die Strömungsströme verschwindet. Vorläufig aber ist der Nachweis von Aktionsströmen an den Körperarterien nicht erbracht.

Eine zweite Versuchsreihe ging von der Annahme aus, dass bei einer peristaltischen Tätigkeit der Arterien eine relative Zunahme der Wandstärke der Arterien im Vergleich zum Durchmesser nach der Peripherie zu erwarten sei. Die hierauf gerichteten Untersuchungen sind zwar noch nicht abgeschlossen, da der Verfasser (W. Heptner) seit Beginn des Krieges im Felde steht, lassen aber schon jetzt mit Bestimmtheit erkennen, dass das Verhältnis von Lumen und Wandstärke im Arteriensystem bis zu Gefässen von $0,1 \mathrm{~mm}$ Durchmesser annähernd konstant bleibt. Ein solches Verhalten ist unter der Voraussetzung zu erwarten, dass der mittlere arterielle Druck bis zu den Kapillaren nicht wesentlich abnimimt. Da aber auch dieser Punkt noch strittig ist, bringen die Versuche vorläufig keinen wesentlichen Beitrag zur Entscheidung unserer Frage. Ähnlich wie die gesamte Wandstärke verhält sich die Muskularis.

Das Hauptbedenken gegen eine peristaltische Welle erblicke ich in dem Mangel einer sichtbaren Bewegung der Arterienwand, nach

1) K. Hürthle, Über pulsatorisch-elektrische Erscheinungen an Arterien. Skand. Arch. f. Physiol. Bd. 29 S. 100. 1913. 
der ich in den Gefässen des Froschmesenteriums durch subjektive Beobachtung gesucht habe. Der mögliche Einwand; dass die Arterien des Frosches sich anders verhalten als die des Säugers, wird dadurch entkräftet, dass auch beim Frosch das dritte Stadium der Adrenalinwirkung in einer dentlich sichtbaren rückläufigen Bewegung des Blutstromes in den kleinen Arterien erzielt werden kann. Die weitere Möglichkeit, dass die peristaltische Bewegung $\mathrm{zu}$ rasch über die Arterie abläuft, um dem Auge bemerklich zu werden, muss dureh kinematographisehe Untersuchungen entschieden werden, wie sie in der folgenden Abhandlung begonnen sind.

Gleichfalls noch nicht abgeschlossen sind Versuche über die Ursache der grösseren Amplitude des Cruralis- gegenüber dem Karotispulse.

Solange nun direkte Beweise für das Vorkommen einer Peristaltik an den Arterien fehlen, dürfen wir die Hypothese nicht als erwiesen betrachten, zumal da die Mitwirkung physikaliseher Faktoren am Verlauf der Stromkurve (Änderung des inneren und äusseren Widerstandes unter dem Einfluss des Druckes) nicht zweifelhaft ist. Die Entscheidung muss daher der Zukunft überlassen werden, und ich erwarte eine Klärung des Frage von der Fortsetzung der Versuche mit künstlichen, abwechselnd mit Blut und Ringer-Lösung erzeugten Pulsen sowie von der Untersuchung der Vorgänge in den kleinsten Arterien, wie sie dureh die in der folgenden Abhandlung beschriebene Methode ermöglicht wird.

\section{Der Stromquotient.}

Zur Charakterisierung der Typen der Strompulse, die bei den Versuchen am Schema an den verschiedenen Querschnitten der Bahn auftreten und in Fig. 2 S. 307 der Abhandlung I schematisiert sind, hat es sich als zweckmässig erwiesen, einen Zahlenbegriff anzuwenden, der als Stromquotient (St-Q) bezeichnet wird und das Verhältnis der diastolischen zur systolischen Stromstärke $\left(\frac{v_{\uparrow}}{v_{\downarrow}}\right)$ darstellt, beide auf Druck- und Zeiteinheit bezogen. Der Wert des Quotienten ändert sich im Verlauf der Bahn von Querschnitt zu Querschnitt in folgender Weise: Am Anfang der Bahn besteht Strömung nur im Abschnitt des steigenden Druckes (der Hahnöffnung), während des sinkenden ist sie gleich Null; $\frac{v_{\uparrow}}{v_{\downarrow}}$ ist also $=\frac{a}{0}=\infty$. 
Am Ende der Bahn, unmittelbar vor dem Widerstand, für welchen das Poiseuille'sche Gesetz gilt, ist die Stromstärke in jedem Zeitabschnitt dem Druck proportional; $\frac{v_{\uparrow}}{v_{\downarrow}}=\frac{a}{a}=1$. Im Verlauf des Schlauches nimmt der Quotient mit der Entferuung des untersuchten Querschnitts vom Anfang der Bahn von $\infty$ auf $1 \mathrm{ab}$, also mit dem Verhältnis des peripheren Windkessels zum zentralen. An demselben Querschnitt wird der St-Q mit wachsender Stromstärke kleiner; doch bewegen sich diese Schwankungen innerhalb viel engerer Grenzen. Man sollte nun erwarten, dass eine Bestimmung des St-Q an den Pulsen des lebenden Tieres nur für den Fall von Bedeutung ist und deutliche Unterschiede ergibt, wenn es sich um eine Charakerisierung der Strompulse im Verlauf der arteriellen Bahn, also um einen Vergleich der Pulse am Anfang und im Verlauf der Aortenbabn und in den grösseren Arterien handelt, dass dagegen in einer und derselben Arterie, zum Beispiel der Cruralis, der St-Q überhaupt keine nennenswerten Änderungen erfahre, weil hier der periphere Windkessel, auch wenn sich seine Dehnbarkeit ändert, im Vergleich zum zentralen (der Aorta) stets verschwindend gering bleibt; man wird also erwarten, dass der St-Q in allen Fällen den Wert 1 nur wenig überschreitet. Diese Vermutung bestätigt sich aber durchaus nicht. Bei den normalen Pulsen hat der St-Q der Cruralis Werte, die meist zwischen 1,5 und 3 schwanken und unter der Wirkung erregender Gefässmittel sich auf $\infty$ erheben; nur in einem Falle nähert sich der St.Q dem Werte 1 , nämlich an den Pulsen gelähmter Gefässe (Abhandlung IV); nur in diesem Falle hat er den theoretisch zu erwartenden Wert. In allen anderen Fällen sind auf Grund der Stromstärke und der Elastizität der Bahn andere, nämlich kleinere Werte zu erwarten. Bei der Adrenalinwirkung liess sich sogar zeigen, dass die Änderung der Elastizität der Bahn, welche zur Erklärung des St-Q angenommen werden müsste, der wirklichen gerade entgegengesetzt gerichtet ist. Daraus geht hervor, dass die Übertragung der Theorie vom Schema auf die Tierpulse unzulässig ist; sie führt hier zu falschen Vorstellungen über die beteiligten Faktoren. Die ausserordentlich starken Schwankungen des St-Q in derselben Arterie können nur aus der Mitwirkung eines Faktors erklärt werden, welcher im Schema der arteriellen Bahn nicht berüeksichtigt ist.

Da aber der St-Q unabhängig von unseren Voraussetzungen ist 
und nur das Verhältnis der diastolischen zur systolisehen Stromstärke ausdrückt, können wir den Begriff doch für den Tierpuls beibehalten. Allerdings hat er hier eine andere, aber nicht weniger bezeichnende Bedeutung: Er bringt zum Ausdruck, dass für die Pulse der gelähmten Gefässe dieselben Faktoren massgebend sind wie im Schema, dass aber schon bei den Pulsen normaler Gefässe ein Faktor mitwirkt, der im Schema nicht berücksichtigt ist, und dass mit dem Ansteigen des St-Q, das mit der Erregung der Gefässe verbunden ist, der fragliche Faktor mehr und mehr an Einfluss gewinnt.

Da die Übertragung der Theorie vom Schema auf die Tierpulse sich als nicht zulässig erwiesen hat, sind wir nicht daran gebunden, die zur Bestimmung des St-Q nötige Trennung des Pulses in zwei Abschnitte im Gipfel der Druckkurve vorzunehmen. Aus den oben (Abhandlung III S. 326) angeführten Gründen verlegen wir die Trennung des Tierpulses in den systolischen und diastolischen Abschnitt in den Beginn der dikrotischen Welle. 